\title{
Charlson Comorbidity Index Score and Risk of Severe Outcome and Death in Danish COVID-19 Patients
}

J Gen Intern Med 35(9):2801-3 DOI: $10.1007 / \mathrm{s} 11606-020-05991-\mathrm{Z}$

(C) Society of General Internal Medicine 2020

\section{INTRODUCTION}

Studies assessing the impact of total comorbidity burden on outcomes of COVID-19 in age- and sex-controlled analyses are lacking. Previous studies identified the following single comorbidities as risk factors for poor COVID-19 outcomes: coronary heart disease, congestive heart failure, cardiac arrhythmia, chronic obstructive pulmonary disease, cancer, and diabetes. ${ }^{1}$ In this nationwide study of Danish COVID-19 patients, we investigated if Charlson Comorbidity Index Score (CCIS) was associated with the risk of severe outcome and death.

\section{METHODS}

In Denmark, all permanent residents are provided a unique personal identification number enabling cross-linkage of complete nationwide healthcare registries. We included all patients diagnosed with COVID-19 until May 3, 2020, using information from the Danish Civil Registration System (holding demographic data) and the Danish National Patient Registry (holding data on all hospital contacts classified according to ICD-10 codes). Data on comorbidities 10 years prior to the date of COVID-19 were identified to calculate the exposure of interest: CCIS of $0,1-2,3-4$, and $>4 .^{2}$ Outcomes of interest were (1) a composite of death, diagnosis of severe acute respiratory syndrome (ICD-10: DB972A), intensive care unit (ICU) admission, and (2) death. Logistic regression models adjusted for sex and age as a continuous variable were employed to associate CCIS $1-2$, CCIS 3-4, and CCIS $>4$ with odds of outcomes. The models were used to estimate the absolute risk of both outcomes according to CCIS groups across ages $40-85$ for both sexes.

Daniel Mølager Christensen and Jarl Emanuel Strange contributed equally to this work

Received May 20, 2020

Revised May 20, 2020

Accepted June 12, 2020

Published online June 24, 2020

\section{RESULTS}

A total of 4480 patients were diagnosed with COVID-19, of which 2911 (65.0\%), $1112(24.8 \%), 288$ (6.4\%), and 169 (3.8\%) had a CCIS of $0,1-2,3-4$, and $>4$, respectively (Table 1). The median age of the total population was 55 years, with the oldest patients in the CCIS $3-4$ group (78 years).

Overall, $17.8 \%$ had severe outcome and $9.3 \%$ died. In the CCIS 0 group, 265 (9.1\%) had severe outcome and $77(2.6 \%)$ died. In the CCIS 1-2, 3-4, and > 4 groups, 330 (29.7\%), 123 $(42.7 \%)$, and $80(47.3 \%)$ had severe outcome and 197 (17.7\%), 85 (29.5\%), and 58 (34.3\%) died, respectively.

The odds of severe COVID-19 were significantly increased in CCIS 1-2 (odds ratio [OR], 1.76 [95\% CI, 1.43 to 2.16]), CCIS 3-4 (OR, 2.36 [95\% CI, 1.74 to 3.18]) and CCIS > 4 (OR, 2.67 [95\% CI, 1.87 to 3.81]) compared with those in CCIS 0 . The odds of death were significantly increased for CCIS 1-2 (OR, 2.13 [95\% CI, 1.57 to 2.90]), CCIS 3-4 (OR, 3.00 [95\% CI, 2.06 to 4.38]), and CCIS > 4 (OR, 3.85 [95\% CI, 2.51 to 5.90]) compared with those for CCIS 0.

The estimated absolute risks of severe COVID-19 and death were increased for CCIS 1-2, 3-4, and > 4 compared with those for CCIS 0 across ages and sexes (Fig. 1).

\section{DISCUSSION}

A Charlson Comorbidity Index Score above 0 was associated with an increased risk of severe COVID-19 and death when controlled for age and sex. This expands upon previous findings of individual comorbidities as independent risk factors for poor COVID-19 outcomes. ${ }^{1}$ Our findings may inform epidemic modeling, public health, and clinical decisions regarding the management of the COVID-19 pandemic.

A limitation of this study was that not all patients had reached a study outcome or recovered by the end of the study period. Additionally, it was not possible to associate the individual comorbidities constituting the CCIS with the outcomes due to lack of power. The Danish healthcare system is free with universal access and its ICU capacity was not exceeded during the study period, ${ }^{3}$ which must be considered when interpreting our findings. More studies are needed to assess the impact of comorbidity combinations on COVID-19 outcomes and to determine if other validated comorbidity scores can predict poor outcomes of COVID-19. 
Table 1 Baseline Characteristics

\begin{tabular}{|c|c|c|c|c|c|}
\hline & CCIS $0(n=2911)$ & CCIS 1-2 $(n=1112)$ & CCIS 3-4 $(n=288)$ & CCIS $>4(n=169)$ & Total $(n=4480)$ \\
\hline Male & $1326(45.6)$ & $559(50.3)$ & $149(51.7)$ & $110(65.1)$ & $2,144(47.9)$ \\
\hline Age [IQR] & $47[35,58]$ & $71[56,81]$ & $78[67,85]$ & $77[69,83]$ & $55[41,72]$ \\
\hline CCIS [IQR] & $0[0,0]$ & $1[1,2]$ & $3[3,4]$ & $6[5,8]$ & $0[0,1]$ \\
\hline \multicolumn{6}{|l|}{ Comorbidities, no. (\%) } \\
\hline Myocardial infarction & $0(0.0)$ & $65(5.8)$ & $40(13.9)$ & $44(26.0)$ & $149(3.3)$ \\
\hline Heart failure & $0(0.0)$ & 77 (6.9) & $66(22.9)$ & $55(32.5)$ & $198(4.4)$ \\
\hline Cerebrovascular disease & $0(0.0)$ & $171(15.4)$ & $85(29.5)$ & $50(29.6)$ & $306(6.8)$ \\
\hline Peripheral vascular disease & $0(0.0)$ & $63(5.7)$ & $52(18.1)$ & $45(26.6)$ & $160(3.6)$ \\
\hline Diabetes with complications & $0(0.0)$ & $38(3.4)$ & $60(20.8)$ & $62(36.7)$ & $160(3.6)$ \\
\hline Diabetes & $0(0.0)$ & $206(18.5)$ & $102(35.4)$ & $88(52.1)$ & $396(8.8)$ \\
\hline Dementia & $0(0.0)$ & $100(9.0)$ & $36(12.5)$ & $20(11.8)$ & $156(3.5)$ \\
\hline Hemi- or paraplegia & $0(0.0)$ & $10(0.9)$ & $5(1.7)$ & $6(3.6)$ & $21(0.5)$ \\
\hline Rheumatic disease & $0(0.0)$ & $71(6.4)$ & $34(11.8)$ & $13(7.7)$ & $118(2.6)$ \\
\hline Peptic ulcer & $0(0.0)$ & $24(2.2)$ & $19(6.6)$ & $18(10.7)$ & $61(1.4)$ \\
\hline COPD & $0(0.0)$ & $306(27.5)$ & $93(32.3)$ & $66(39.1)$ & $465(10.4)$ \\
\hline Chronic renal disease & $0(0.0)$ & $30(2.7)$ & $57(19.8)$ & $67(39.6)$ & $154(3.4)$ \\
\hline Mild liver disease & $0(0.0)$ & $35(3.1)$ & $11(3.8)$ & $16(9.5)$ & $62(1.4)$ \\
\hline Severe liver disease & $0(0.0)$ & $0(0.0)$ & $8(2.8)$ & $10(5.9)$ & $18(0.4)$ \\
\hline Cancer & $0(0.0)$ & $156(14.0)$ & $106(36.8)$ & $86(50.9)$ & $348(7.8)$ \\
\hline Metastatic cancer & $0(0.0)$ & $0(0.0)$ & $0(0.0)$ & $49(29.0)$ & 49 (1.1) \\
\hline HIV/AIDS & $0(0.0)$ & $0(0.0)$ & $0(0.0)$ & * & * \\
\hline
\end{tabular}

CCIS, Charlson comorbidity index score; COPD, chronic obstructive pulmonary disease; IQR, interquartile range. *Low number of observations, blanked to ensure patient anonymity
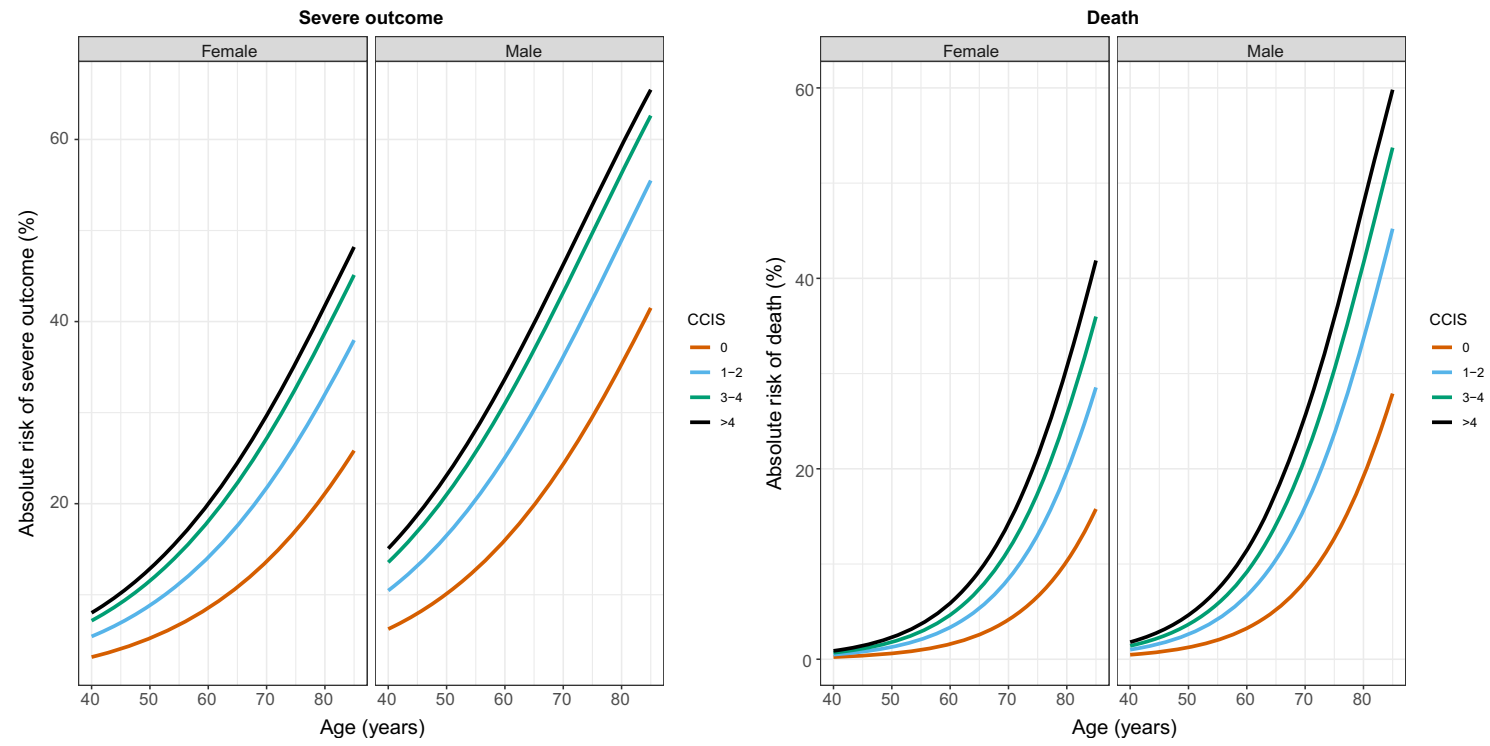

Figure 1 Estimated risks of severe outcome and death according to sex, age, and Charlson Comorbidity Index Score (CCIS). 
Daniel Mølager Christensen, $M D^{1}$

Jarl Emanuel Strange, $M D^{2}$

Gunnar Gislason, $\mathrm{MD}, \mathrm{PhD}^{1}$

Christian Torp-Pedersen, MD, DMSc ${ }^{3}$

Thomas Gerds, dr.rer.nat ${ }^{4}$

Emil Fosbøl, $M D, P h D^{5}$

Matthew Phelps, $M P H, P h D^{1}$

${ }^{1}$ The Danish Heart Foundation,

Copenhagen, Denmark

${ }^{2}$ Department of Cardiology, Gentofte University Hospital,

Copenhagen, Denmark

${ }^{3}$ Department of Clinical Research, Nordsjaellands Hospital,

Hillerød, Denmark

${ }^{4}$ Department of Biostatistics, University of Copenhagen,

Copenhagen, Denmark

${ }^{5}$ Department of Cardiology, Rigshospitalet,

Copenhagen, Denmark
Corresponding Author: Daniel Mølager Christensen, MD; The Danish Heart Foundation Copenhagen, Denmark (e-mail: dmchristensen@hjerteforeningen.dk).

\section{Compliance with Ethical Standards:}

Conflict of Interest: Dr. Torp-Pedersen reports grants from Bayer and grants from Novo Nordisk outside the submitted work; all other authors reported no conflicts of interest.

\section{REFERENCES}

1. Guan W-J, Liang W-H, Zhao Y, et al. Comorbidity and its impact on 1590 patients with Covid-19 in China: A Nationwide Analysis. Eur Respir J. 2020; Available from: https://doi.org/10.1183/13993003.00547-2020

2. Guan $\mathbf{H}$, Sundararajan V, Halfon $\mathbf{P}$, et al. Coding algorithms for defining comorbidities in ICD-9-CM and ICD-10 administrative data. Med Care. 2005;43(11):1130-9.

3. Statens Serum Institut. COVID-19 in Denmark Epidemiological Surveillance Report (Danish) [Internet]. 2020 [cited 2020 Apr 24]. Available from: https://files.ssi.dk/COVID19-overvaagningsrapport-24042020-ds65

Publisher's Note: Springer Nature remains neutral with regard to jurisdictional claims in published maps and institutional affiliations. 